\title{
Effects of omalizumab treatment on serum cytokine concentrations of atopic patients with chronic spontaneous urticaria: a preliminary report
}

\author{
${ }^{1}$ Division of Internal Medicine, Allergy and Clinical Immunology, Department of Medical and Surgical Sciences, Second \\ University of Naples School of Medicine, Naples, Italy \\ ${ }^{2}$ Allergy Clinic, "F. Magrassi - A. Lanzara" Department of Clinical and Experimental Medicine and Surgery, Second Univer- \\ sity of Naples School of Medicine, Naples, Italy \\ ${ }^{3}$ Current address: "S. Carlo" Hospital, Potenza, Italy \\ ${ }^{4}$ Current address: Clinic Center, Naples, Italy
}

\section{KEY wORDS}

omalizumab; chronic spontaneous urticaria; cytokines; Th1; Th2; atopy

\author{
Corresponding author \\ Ciro Romano \\ Division of Internal Medicine, \\ Allergy and Clinical Immunology \\ Second University of Naples School of \\ Medicine \\ Piazza Luigi Miraglia, 3 \\ 80131 Naples, Italy \\ Phone: +390815665028 \\ Fax: +390815665080 \\ E-mail: ciro.romano@unicampania.it \\ Doi \\ 10.23822/EurAnnACI.1764-1489.10
}

\begin{abstract}
Summary
Omalizumab has recently obtained indication for chronic spontaneous urticaria both in the US and Europe. However, the mechanism of action of this drug has yet to be fully elucidated. Previous studies have shown elevations in cytokine serum levels in patients with chronic spontaneous urticaria, and it is not known whether omalizumab treatment may affect cytokine serum levels in this condition. Besides, a proportion of chronic spontaneous urticaria patients have concomitant atopy, which may be associated, at least in theory, with prevalence of serum Th2-type cytokines. In this study, serial serum samples from five patients (4 atopic and 1 nonatopic) with chronic spontaneous urticaria were assayed for cytokine concentrations by means of flow-cytometry-based multiplex bead assays, before and during omalizumab treatment. Omalizumab appeared to significantly affect the concentrations of multiple cytokines in a case of severe, long-lasting chronic spontaneous urticaria. Interestingly, IL-22 serum levels were found to progressively increase in three of five patients. Further studies are thus needed in larger patient populations, to conclusively establish whether the mechanism of action of omalizumab in chronic spontaneous urticaria also includes modulation of cytokine synthesis.
\end{abstract}

\section{Introduction}

Omalizumab, a humanized monoclonal antibody targeting human IgE, was originally designed for therapeutic use in moderate to severe allergic asthma (1). To put it simply, by binding circulating free $\mathrm{IgE}$, omalizumab prevents mast cell degranulation and downstream mediator effects (2). Since omalizumab has been suggested to be possibly beneficial for management of chronic spontaneous urticaria as well (3-5), randomized placebo-controlled trials have been carried out to assess efficacy of
anti-IgE therapy in chronic spontaneous urticaria $(6,7)$, culminating in its recent approval for this indication both in the U.S. and Europe. However, despite robust evidence on omalizumab effectiveness in chronic spontaneous urticaria, how this monoclonal antibody works in this setting is not yet fully understood. Here, we report further data on the pleiotropic effects of omalizumab in chronic spontaneous urticaria, and specifically on its effects on cytokine serum levels in a small series of patients, most of whom with concomitant atopy. 


\section{Patients, materials, and methods}

\section{Patients}

Since 2008, among the patients suffering from chronic spontaneous urticaria resistant to standard therapy who agreed to undergo off-label treatment with omalizumab, five $(1 \mathrm{M}, 4 \mathrm{~F}$; mean age $\pm S D /$ median: $52.6 \pm 13.5 / 52$ ) consented to donate a venous blood sample before initiating anti-IgE treatment, and prior to each subsequent injection. Diagnosis of chronic spontaneous urticaria was made according to international guidelines (8). Four out of five patients were found to be atopic, with sensitization to airborne allergens (mainly grass pollen and wall pellitory). Mean $\pm S D /$ median duration of disease was $48.4 \pm$ $61.9 / 36$ months. Mean $\pm \mathrm{SD} /$ median total serum IgE were 215.3 $\pm 206.9 / 192 \mathrm{kU} / \mathrm{L}$. Patients tested negative to autologous serum skin test (ASST) or basophil histamine release assay (BHRA). Since patients were selected based only on their willingness to donate a blood sample before omalizumab treatment and at each visit thereafter, the frequency of atopy and ASST status was just a coincidental result. Patients were only allowed to take antihistamines in the week preceding the start of omalizumab therapy, in order to avoid steroids affecting serum cytokine concentrations. All patients were strictly monitored during omalizumab treatment, as previously detailed (9). Complete response was defined as complete resolution of symptoms and signs of disease; partial response was defined as a significant reduction in the urticaria activity score over 7 days (UAS7); no response was considered in case of no benefit at all (9). All patients gave their informed consent prior to beginning off-label omalizumab treatment. Finally, five healthy nonatopic subjects recruited from hospital staff donated a single blood sample at the beginning of the study, in order to compare baseline serum cytokine concentrations between chronic spontaneous urticaria patients and healthy controls. The study was approved by the institutional ethics committee.

\section{Serum cytokine concentration measurement}

Patient sera were stored at $-20^{\circ} \mathrm{C}$ until use. Serum cytokine levels were measured using a flow cytometry-based multiplex bead array system. The FlowCytomix human Th1 / Th2 (Bender MedSystems, Vienna, Austria) and the FlowCytomix human Th1 / Th2 / Th9 / Th17 / Th22 (eBioscience, San Diego, CA, USA) kits were used, according to the manufacturers' instructions. The following cytokines were assayed: IL-12p70, interferon- $\gamma$, IL-17A, IL-2, IL10, IL-9, IL-22, IL-6, IL-13, IL-4, IL-5, IL-8, IL-1 $\beta$, TNF- $\alpha$.

\section{Omalizumab schedule}

Because of no clear indications as to how to treat chronic spontaneous urticaria patients at the time of enrollment, the sched- ule proposed for allergic asthma, i.e., based on body weight and total serum IgE concentrations, was adopted. Omalizumab dose was increased in case of unsatisfactory response or slowly tapered off in case of remission, as previously detailed $(5,9)$.

\section{Results}

\section{Patients}

Four out of the five patients treated with omalizumab had a complete remission (3 with concomitant atopy, plus the only nonatopic patient); the remaining atopic patient did not benefit at all from anti-IgE therapy. Complete response was achieved within a few days in most of the patients.

\section{Serum cytokine concentrations}

Most patients had detectable levels of a few cytokines; only one patient had elevated levels of multiple cytokines. This patient had the most severe form of chronic spontaneous

Table 1 - Behavior of cytokine serum levels in chronic spontaneous urticaria patients following omalizumab treatment.

\begin{tabular}{ccc}
\hline Cytokine & Patient code & Effect \\
\hline IL-12p70 & AM1 & increase \\
\hline IFN- $\boldsymbol{\text { ZR5 }}$ & reduction \\
\hline IL-17A & AM1 & reduction \\
\hline IL-2 & ZR5 & stable \\
\hline IL-9 & AM1 & stable \\
\hline & AM1 & stable \\
\hline IL-22 & GR3 & stable \\
\hline & ZR5 & stable \\
\hline & PM4 & increase \\
\hline IL-13 & ZR5 & increase \\
\hline & GR3 & increase \\
\hline PM4 & stable \\
\hline AM1 & stable \\
\hline TNF- $\alpha$ & ZR5 & stable \\
\hline IL-4 & GR3 & stable \\
\hline
\end{tabular}

Not mentioned cytokines were undetectable. Patient SF2, who was the only nonatopic subject, did not display any detectable cytokine. 
Figure 1 - Effects of omalizumab treatment on multiple serum cytokine levels in the patient with the most severe form of chronic spontaneous urticaria (giant wheals can be seen in the inset). Time is expressed as weeks ( $w$ ) of treatment; T0: before start of omalizumab treatment.
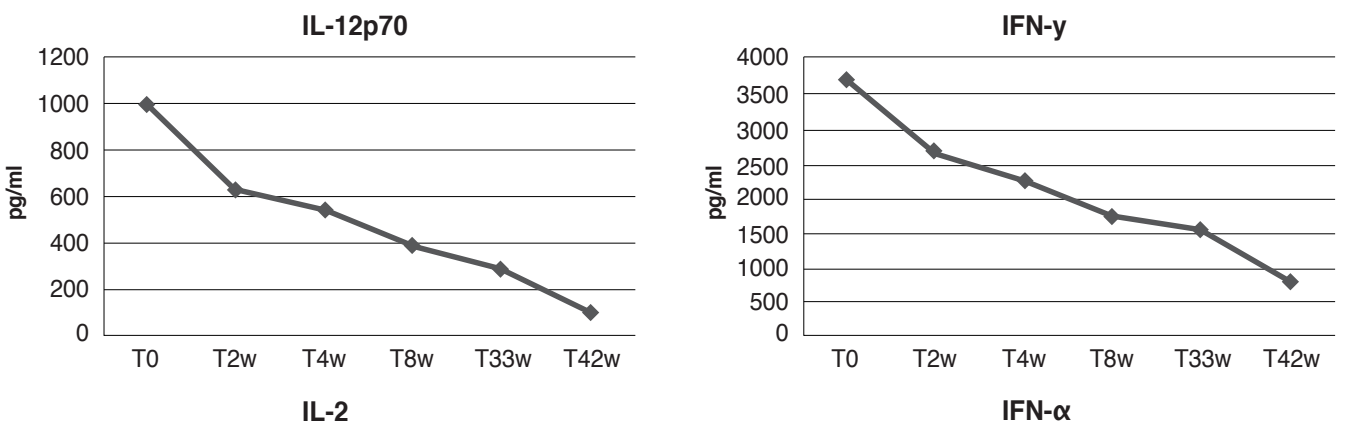

Patient code: ZR5
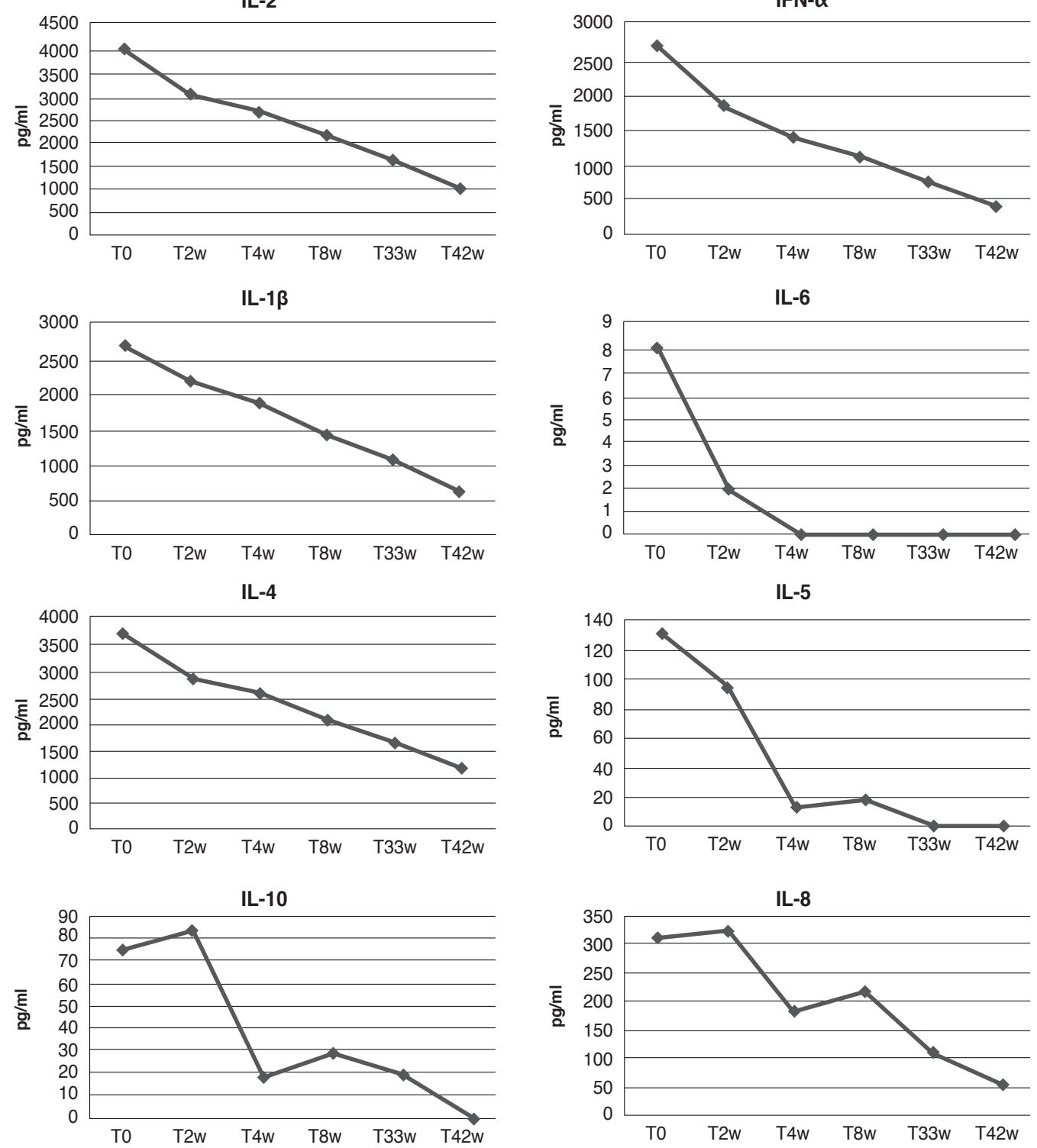


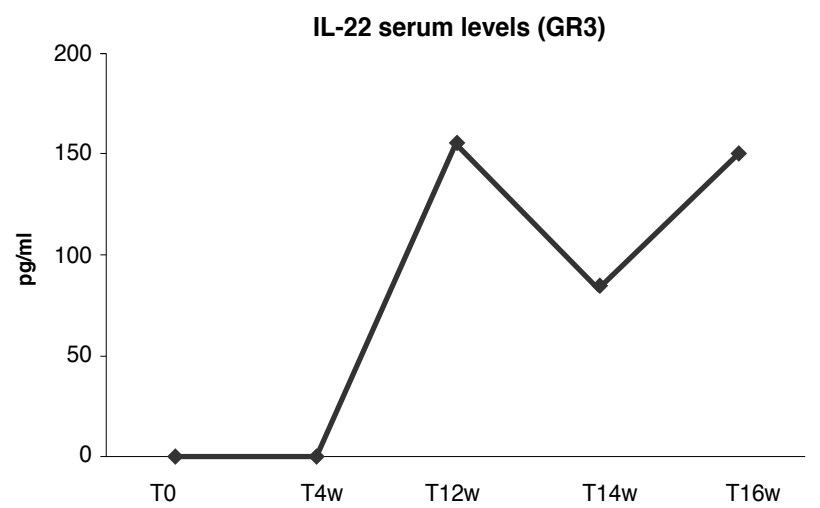

IL-22 serum levels (PM4)

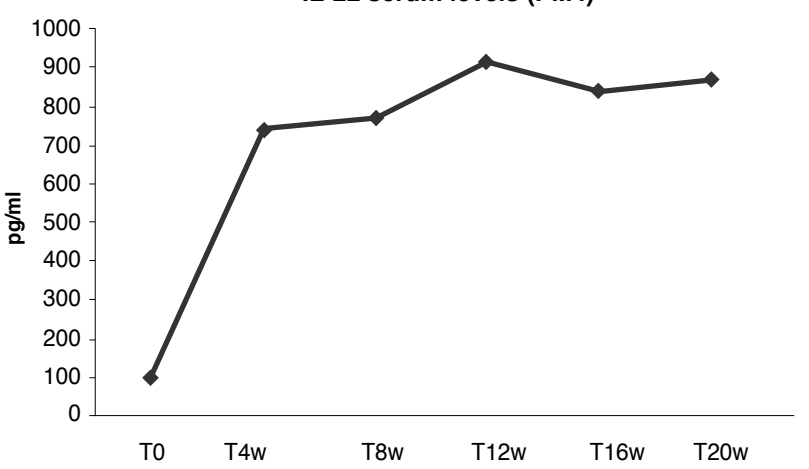

urticaria with giant wheals before treatment (figure 1), and a decade-long history of disease. Interestingly, omalizumab determined a progressive reduction in cytokine serum levels with time (figure 1). Of note, Th2-type cytokines such as IL13 and IL-9 were not shown to be influenced by omalizumab treatment; three patients showed a progressive elevation in IL-22 serum levels (figure 2 ). The remaining cytokines were not detectable. Interestingly, the only nonatopic patient did not have any measurable cytokine in serum. Serum cytokines were undetectable in the five healthy subjects as well. Table 1 summarizes the behavior of cytokine serum levels in our patients.

\section{Discussion}

Omalizumab now represents a further advancement in the treatment of chronic spontaneous urticaria, due to its recent approval for this indication by the FDA and the EMEA. Although omalizumab efficacy in chronic spontaneous urticaria is unquestionable, its mechanism of action is still incompletely understood. Downregulation of high affinity $\operatorname{IgE}$ receptors (FceRI) on mast cells, as a consequence of circulating $\mathrm{IgE}$
Figure 2 - Behavior of IL-22 serum levels in three of the five patients treated with omalizumab. IL-22 serum levels increased independently of clinical response (PM4 and ZR5 had both a complete response, GR3 was a nonresponder). Time is expressed as weeks ( $w$ ) of treatment; TO: before start of omalizumab treatment.

\section{IL-22 serum levels (ZR5)}

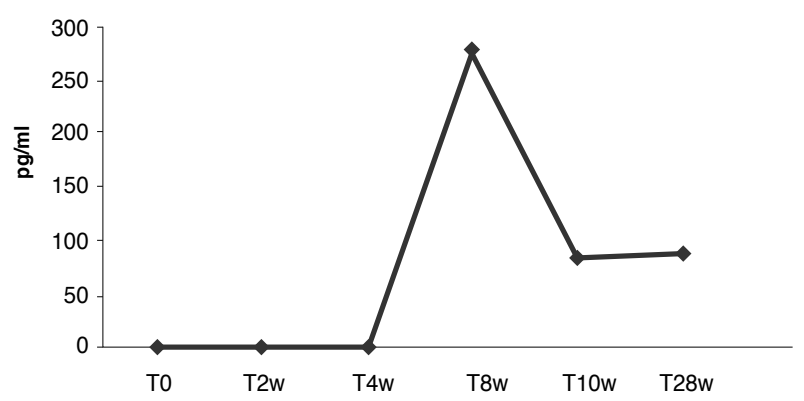

neutralization, was initially deemed to be the main mechanism underlying efficacy of omalizumab in this peculiar dermatologic condition (10). In addition, because IgE binding to FceRI has been demonstrated to favor proliferation and survival of mast cells and to lower the threshold for mediator release (11), unavailability of $\mathrm{IgE}$ due to clearance by omalizumab obviously has negative consequences on these aspects of mast cell biology. When also taking into account clinical observation, hints for additional mechanisms of action can be deduced by the very rapid onset of action, effectiveness maintained well beyond the drug half-life and/or with suboptimal doses for complete $\operatorname{IgE}$ neutralization, and evidence of no inhibition of mast cell ability to degranulate in vivo $(5,9)$. Besides, unlike with asthma, to achieve remission in chronic spontaneous urticaria, omalizumab dose and administration schedule need not to be calculated based on body weight and serum total IgE concentration (6,7). Taken together, all these observations indicate a more complex mechanism of action for omalizumab than initially theorized.

In this study, we retrospectively assayed longitudinal serum samples from patients with chronic spontaneous urticaria for cytokine content, before and during omalizumab treat- 
ment. The majority of patients ( 4 out of 5) were also atopic. Reports on cytokine serum levels in chronic spontaneous urticaria patients are not numerous in the literature. Most of the studies have emphasized the observation of elevated levels of proinflammatory cytokines and their relationship with disease severity (12-14). Conversely, scant evidence exists with regard to Th2-type cytokines (15). In addition, only a few cytokines in a given patient population have been evaluated in the studies available in the literature (12-15). On the contrary, we have retrospectively measured the serum levels of a large number of cytokines in our patients before and during omalizumab treatment, taking advantage of multiplex bead-based flow cytometric assays, which allow simultaneous determination of multiple cytokines in a single laboratory session.

A striking reduction in cytokine serum levels was observed with time in the patient with the most severe form of chronic spontaneous urticaria, who rapidly obtained a complete response. In the remaining patients, chronic spontaneous urticaria tended to manifest with less severity, which may explain, at least in part, the different pattern of serum cytokine serum levels.

Overall, omalizumab did not appear to affect IL-9 and IL13 serum levels; only one patient showed undetectable levels of all serum cytokines investigated; interestingly, this patient was the only nonatopic subject, and his cytokine serum levels were indistinguishable from those of healthy subjects. Finally, IL-22 serum levels became detectable following institution of omalizumab therapy in three patients; two of these patients were complete responders and one was a nonresponder. Thus, this effect appeared to be due to omalizumab treatment irrespective of the clinical response. Since a recognized function of Th22 cells is to protect epithelial barrier organs including the skin, this effect may theoretically be interpreted as beneficial (16). However, definitive conclusions cannot be drawn at this time, due to the small number of patients analyzed thus far, and to the lack of homogeneous, consistent categories of patients.

\section{Conclusions}

Omalizumab treatment appeared to affect cytokine serum levels in atopic patients with chronic spontaneous urticaria. Whether this effect may be relevant to its mechanism of action in chronic spontaneous urticaria remains to be established in future studies with larger numbers of patients, possibly including atopic, nonatopic, and autoimmune urticaria subjects.

\section{References}

1. Humbert N, Beasley R, Ayres J, et al. Benefits of omalizumab as add-on therapy in patients with severe persistent asthma who are inadequately controlled despite best available therapy (GINA 2002 step 4 treatment): INNOVATE. Allergy. 2005; 60(3):309-16.

2. Chang TW, Wu PC, Hsu CL, Hung AF. Anti-IgE antibodies for the treatment of IgE-mediated allergic diseases. Adv Immunol. 2007;93:63-119.

3. Spector SL, Tan RA. Effect of omalizumab on patients with chronic urticaria. Ann Allergy Asthma Immunol. 2007;99(2):190-3.

4. Kaplan AP, Joseph K, Maykut RJ, Geba GP, Zeldin RK. Treatment of chronic autoimmune urticaria with omalizumab. J Allergy Clin Immunol. 2008;122(3):569-73.

5. Romano C, Sellitto A, De Fanis U, et al. Maintenance of remission with low-dose omalizumab in long-lasting, refractory chronic urticaria. Ann Allergy Asthma Immunol. 2010;104(1):95-7.

6. Saini S, Rosen KE, Hsieh HJ, et al. A randomized, placebo-controlled, dose-ranging study of single-dose omalizumab in patients with $\mathrm{H} 1$-antihistamine-refractory chronic idiopathic urticaria. J Allergy Clin Immunol. 2011;128(3):567-73.

7. Maurer M, Rosen K, Hsieh HJ, et al. Omalizumab for the treatment of chronic idiopathic or spontaneous urticaria. N Engl J Med. 2013;368(10):924-35.

8. Zuberbier T, Aberer W, Asero R, et al. The EAACI/GA(2)LEN/ $\mathrm{EDF} / \mathrm{WAO}$ Guideline for the definition, classification, diagnosis, and management of urticaria: the 2013 revision and update. Allergy. 2014;69(7):868-87.

9. Romano C, Sellitto A, De Fanis U, et al. Omalizumab for difficult-to-treat dermatological conditions: clinical and immunological features from a retrospective real-life experience. Clin Drug Investig. 2015;35(3):159-68.

10. Saavedra MC, Sur S. Down regulation of the high-affinity IgE receptor associated with successful treatment of chronic idiopathic urticaria with omalizumab. Clin Mol Allergy. 2011;9(1):2.

11. Asai K, Kitaura J, Kawakami Y, et al. Regulation of mast cell survival by IgE. Immunity. 2001;14(6):791-800.

12. Dos Santos JC, Azor MH, Nojima VY, et al. Increased circulating pro-inflammatory cytokines and imbalanced regulatory $\mathrm{T}$-cell cytokines production in chronic idiopathic urticaria. Int Immunopharmacol. 2008;8(10):1433-40.

13. Atwa MA, Emara AS, Youssef N, Bayoumy NM. Serum concentrations of IL-17, IL-23 and TNF-a among patients with chronic spontaneous urticaria: association with disease activity and autologous serum skin test. J Eur Acad Dermatol Venereol. 2014;28(4):469-74.

14. Rasool R, Ashiq I, Shera IA, Yousuf Q, Shah ZA. Study of serum interleukin (IL) 18 and IL-6 levels in relation with the clinical disease severity in chronic idiopathic urticaria patients of Kashmir (North India). Asia Pac Allergy. 2014;4(4):206-11.

15. Daschner A, Rodero M, DE Frutos C, et al. Different serum cytokine levels in chronic vs. acute Anisakis simplex sensitization-associated urticaria. Parasite Immunol. 2011;33(6):357-62.

16. Eyerich K, Eyerich S. Th22 cells in allergic disease. Allergo J Int. 2015;24(1):1-7. 